\title{
On the homoclinic orbits of the Lü system
}

\author{
M. Alvarez-Ramírez \\ Departamento de Matemáticas UAM-Iztapalapa, \\ San Rafael Atlixco 186, Col. Vicentina, \\ 09340 Iztapalapa, México city, México \\ mar@xanum.uam.mx \\ J. D. García-Saldaña \\ Departamento de Matemática y Física Aplicadas, \\ Facultad de Ingeniería, \\ Universidad Católica de la Santísima Concepción, \\ Casilla 297, Concepción, Chile \\ jgarcias@ucsc.cl
}

Received (to be inserted by publisher)

\begin{abstract}
In this paper the existence of homoclinic orbits of the equilibrium point $(0,0,0)$ is demonstrated in the case of the of Lü system for parameter values did not report by G. A. Leonov. In addition, some simulations are shown that agree with our theoretical analysis.
\end{abstract}

Keywords: Lü system, chaotic, homoclinic orbit, bifurcation

\section{Introduction}

In this paper we consider the Lü system [Lü \& Chen , 2002]

$$
\begin{aligned}
& \dot{x}=a(y-x), \\
& \dot{y}=c y-x z, \\
& \dot{z}=-b z+x y
\end{aligned}
$$

where $a, b, c \in \mathbb{R}^{+}$are parameters.

For the parameter set given by $a=36, b=3$ and $c=20$ this system (1) is a chaotic system which bridges the gap between the Lorenz and the Chen attractors [Lü \& Chen , 2002]. They all have the same symmetry, stability of equilibrium points, and similar bifurcations and topological structures, in among other things [Lü \& et. al. , 2002].

In the study of the chaotic systems a particular role is played by the homoclinic orbits because the existence of these suggests the existence of chaotic dynamics, see [Guckenheimer \& Holmes , 1983]. The system (1) has a saddle point at $(0,0,0)$, for all positive parameter values. This paper concerned with the problem of finding conditions to ensure the existence of homoclinic orbits (joins the saddle equilibrium point to itself) of Lü system at origin.

Since the introduction of the Lü system, several articles related to this system of equations have been Dublished, see for instance [Leonov, 2012], [Leonov , 2013], [Leonov \& Kuznetsov , 2015], [Leonov , 2016] sind $\stackrel{9}{9}$ eferences therein. One central aspect concerns to the existence of homoclinic orbits related to $(0,0,0)$. 
Recently, Tigan \& Llibre [2016] were able to apply results by Belykh [1984] to the Chen system and they showed the existence of homoclinic orbits of the saddle point. In this paper, we show that the same technique can be applied to the Lü system.

\section{Equilibrium points and their stability}

The system (1) is invariant under the transformation $S(x, y, z)=(-x,-y, z)$. One easily verifies that the system (1) has three equilibrium points, $E_{0}=(0,0,0)$, which exists for any parameter values and a pair of symmetrically located equilibria $E_{+}=(\sqrt{b c}, \sqrt{b c}, c)$ and $E_{-}=(-\sqrt{b c},-\sqrt{b c}, c)$ in the case that $b c>0$.

Lemma 1. If parameters $a, b$ and $c$ are positive, then the following statements about system (1) hold.

(i) The equilibrium point $(0,0,0)$ is a saddle point.

(ii) For $c<\frac{a+b}{3}$, the equilibrium points $E_{+}$and $E_{-}$are stable.

Proof. The linearization of the vector field associated to (1) about an equilibrium point is given by the Jacobian matrix

$$
A=\left(\begin{array}{rrr}
-a & a & 0 \\
-z & c & -x \\
y & x & -b
\end{array}\right) .
$$

It easy to see that at $(0,0,0)$ the linearization matrix has two negative eigenvalues $\lambda_{1}=-a$ and $\lambda_{2}=-b$, while the other one is positive, $\lambda_{3}=c$, so this equilibrium is unstable.

The linearization of (1) at the equilibrium points $E_{ \pm}$has the characteristic polynomial given by

$$
p(\lambda)=\lambda^{3}+(a+b-c) \lambda^{2}+a b \lambda+2 a b c=0 .
$$

As a consequence of the Routh-Hurwitz criterion we get that all of the roots of $p(\lambda)$ are negative or have negative real part if only if

$$
a+b-c>0, \quad 2 a b c>0 \text { and } a+b-c>2 c .
$$

So, all the roots have negative real parts when $0<c<\frac{a+b}{3}$. The lemma is thus proved.

Since the equilibrium point at the origin is a saddle, then there are two invariant manifolds associated to it. A stable manifold $W^{s}$, which is two-dimensional and a one-dimensional unstable manifold $W^{u}$. This unstable manifold consists of $(0,0,0)$ and a pair of orbits (separatrices), which are symmetrical to each other under the symmetry $S(x, y, z) \rightarrow(-x,-y, z)$.

Linearization of the system at $(0,0,0)$ yields that the tangent stable space $T W_{0}^{s}$ is given by

$$
T W^{s}=\{y+z=0\},
$$

and for the tangent unstable space $T W_{0}^{u}$,

$$
T W^{u}=\left\{y+\frac{a}{a+c} x=0, z=0\right\} .
$$

\section{Existence of homoclinic orbits}

In this section we transform the system (1) into a set of new equations which have already been studied by Belykh [1984] and then show the existence of the separatrices loops of the saddle point.

Firstly, we recall that Zhang \& et. al. [2012] proved that by assuming $2 a>b>2 c>0$ and taking a Lyapunov-like function of the form

$$
V(x, y, z)=k_{1} x^{2}+\left(y-k_{2} x\right)^{2}+(z-\tau)^{2}
$$

where $k_{1}>0, \frac{b}{2 a}>k_{2}>\frac{c}{a}$ and $\tau=a k_{1}+a k_{2}-c k_{2}+a k_{2}^{2}$ the solutions of the Lü system (1) are globally bounded for all positive time. 
Here we are considering the case $2 a>b>0$ and $a>c>0$. By non-linear space change of coordinates

$$
u=\frac{x}{\sqrt{2}}, \quad v=\frac{a(y-x)}{\sqrt{2}}, \quad w=-\frac{x^{2}}{2}+a z
$$

the system (1) can be reduced to the form

$$
\begin{aligned}
\dot{u} & =v, \\
\dot{v} & =a c u-u^{3}+(c-a) v-u w, \\
\dot{w} & =(2 a-b) u^{2}-b w .
\end{aligned}
$$

Observe that the spatial transformation (3) is similar to the one used by Tigan \& Llibre [2016] when they study the existence of homoclinic orbits in the Chen system. However, the transformation used here is simpler than the one adopted in this last reference since they use also a time reparametrization added to the spatial transformation.

Moreover, if we take $a c=1, \delta=2 a-b>0, \lambda=a-c>0$ and $\sigma=b$, then system (4) becomes

$$
\begin{aligned}
\dot{u} & =v, \\
\dot{v} & =-\left(u^{2}+w-1\right) u-\lambda v, \\
\dot{w} & =-\sigma w+\delta u^{2} .
\end{aligned}
$$

This corresponds to the system already studied in [Belykh , 1984]. Therefore, we obtain the following theorem.

Theorem 1. For each $\sigma>0$, there is, in the region of positive parameters $\delta$ and $\lambda$, a bifurcation curve $\{\rho(\sigma, \delta, \lambda)=0\}$, beginning at $(0,0)$ and going to infinity for $\delta \rightarrow \infty$, corresponding to a homoclinic orbit of the saddle point $(0,0,0)$ of the system (1).

The statement means that for any set of parameters $(a, b, c) \in\left(\mathbb{R}^{+}\right)^{3}$ for which $a c=1,2 a>b>0$ and $a>c>0$, the Lü system has two symmetrical homoclinic orbits to the origin equilibrium point.

In addition to this, we emphasize that Leonov [2013] considered the linear scaling, $a \neq 0$,

$$
a \rightarrow 1, \quad b \rightarrow \frac{b}{a}, \quad c \rightarrow \frac{c}{a}
$$

when studying Lü and Chen systems, such that the system (1) depends only on two parameters, namely $\tilde{b}=b / a$ and $\tilde{c}=c / a$ and, by using the Fishing principle (see [Leonov, 2012]), he was able to prove this result.

Theorem 2. Let $a=1$ and $\tilde{c} \in(0.101,1)$. Then there exits $\tilde{b} \in(0,2)$ such that the system (1) has a homoclinic orbit of the saddle $x=y=z=0$.

It is worth to observe that under the conditions set in Theorem 2, it follows that $2-b / a>0$ and $1-c / a>0$. Therefore, Theorem 1 is taking into account the case $a c=1$ not considered in Theorem 2 , obtaining a more complete picture for the Lü system.

According to Theorem 1, Lü system admits homoclinic orbits if the values of the parameters satisfy $a c=1,2 a-b>0$ and $a-c>0$, so it means that $a>1,0<b<2$ and $0<c<1$. In order to check out numerically our theoretical results, we were chosen the initial conditions very close to the origin.

We have taken for our computation two set of parameter values given by $(a, b, c)=$ $(1.219814363,1.083333333,1 / a)$ and $(a, b, c)=(1.384785404,0.684210526,1 / a)$ satisfying the initial condition $x_{0}=0.000001, z_{0}=0$ and $y_{0}$ on the local 1-dimensional unstable manifold, that is, $y_{0}=-\frac{a}{a+c} x_{0}$. The results are depicted in Figure 1.

\section{Conclusion}

The existence of homoclinic orbits of the Lü system has been examined with special attention to the parameter region with $a c=1,2 a-b>0$ and $a-c>0$. To achieve this target we use a method based 

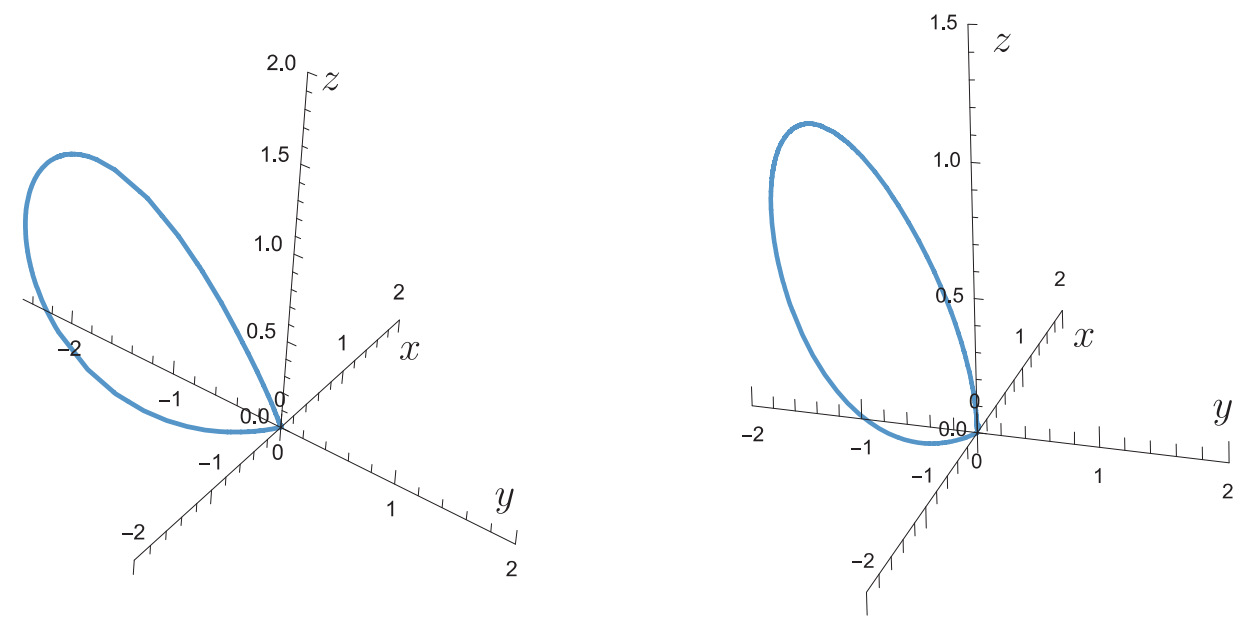

Fig. 1. Numerical computation of homoclinic orbits view in $x y z$ coordinates where values of the parameters shown are, from left to right, $(a, b, c)=(1.219814363,1.083333333,1 / a)$ and $(a, b, c)=(1.384785404,0.684210526,1 / a)$, respectively.

on comparison of differential equation systems given in [Belykh, 1984]. We found that Lü system has homoclinic orbits at $(0,0,0)$ for some parameter values, which are cases not covered in [Leonov, 2012]. We perform numerical experiments to verify our theoretical results. The simulation results were produced using Mathematica(R) program.

\section{Acknowledgments}

M. Alvarez-Ramírez was partially supported by the grant Red de cuerpos académicos Ecuaciones Diferenciales. Proyecto sistemas dinámicos y estabilización. PROMEP 2011-SEP, Mexico; and J. D. García-Saldaña was supported by FONDECyT postdoctoral fellowship 3150131/2015.

\section{References}

Belykh, V. N. [1984] "Bifurcations of separatrices of a saddle point of the Lorenz system," Diff. Eqs. 20, 1184-1191.

Chen, G. \& Ueta, T. [1999] "Yet another chaotic attractor," Int. J. Bifurcation and Chaos 9 (7), $1465-1466$.

Guckenheimer, J. \& Holmes, P. [1983] Nonlinear Oscillations, Dynamical Systems and Bifurcations of Vector Fields, Applied Math. Sciences, Vol. 42 (Springer-Verlag, NY).

Leonov, G. A. [2012] "General existence conditions of homoclinic trajectories in dissipative systems. Lorenz, Shimizu-Morioka, Lu and Chen systems," Physics Letters A 376, 3045-3050.

Leonov, G. A. [2013] "Criteria for the existence of homoclinic orbits of systems Lü and Chen," Doklady Mathematics 87, 220-223.

Leonov, G. A. [2016] "Necessary and sufficient conditions of the existence of homoclinic trajectories and cascade of bifurcations in Lorenz-like systems: birth of strange attractor and 9 homoclinic bifurcations," Nonlinear Dynam. 84, 1055-1062.

Leonov, G. A. \& Kuznetsov, N. V. [2015] "On differences and similarities in the analysis of Lorenz, Chen, and Lu systems," Appl. Math. Comput. 256 , 334-343.

Lü, J. \& Chen, G. [2002] "A new chaotic attractor coined," Int. J. Bifurcation and Chaos 12, 1789-1812.

Lü, J., Chen, G., Cheng, D. \& Celikovsky, S. [2002] "Bridge the gap between the Lorenz system and the Chen system," Int. J. Bifurcation and Chaos 12 , 2917-2926.

Tigan, G. \& Llibre, J. [2016] "Heteroclinic, homoclinic and closed orbits in the Chen system," Int. J. Bifurcation and Chaos 26, 1650072.

Zhang, F., Mu, C. \& Li, X. [2012] "On the boundness of some solutions of the Lü system," Int. J. Bifurcation and Chaos 22, 1-5. 